\title{
Consistent Approximations to Impulsive Optimal Control Problem
}

Daniella Porto ${ }^{1}$

Geraldo Nunes Silva ${ }^{2}$

Heloísa Helena Marino Silva ${ }^{3}$

Departamento de Matemática Aplicada, Universidade Estadual Paulista-Unesp, São José do Rio Preto, SP

\begin{abstract}
We study impulsive optimal control problem and apply a theory called consistent approximations which was introduced in $[1,2]$. From an infinite dimension problem $(P)$, we can build a sequence of discrete problems $\left(P_{N}\right)$ with finite dimension. We show that these discrete problems epi-converge to $(P)$ which ensures that all sequence of global or local minimum of $\left(P_{N}\right)$ that converge, will converge to a global or local minimum of $(P)$, respectively.
\end{abstract}

Key-words. Impulsive Optimal Control Problem, Consistent Approximations, Euler's Discretization

\section{Introduction}

There exist many papers that discuss optimality conditions for impulsive optimal control problems where the control systems involve measures. On the other hand, the literature about numerical methods for impulsive optimal control problems is rather scarce.

We show that an impulsive optimal control problem can be discretized by Euler's method to generate a subsequence of optimal trajectories of Euler that converges to an optimal trajectory of the continuous problem, using an appropriate metric.

\section{Theory of Consistent Approximations}

Let $B$ be a normed space. Consider the problem

$$
\text { (P) } \min _{x \in S_{C}} f(x),
$$

where $f: B \rightarrow \mathbb{R}$ is continuous and $S_{C} \subset B$.

Let $\mathcal{N}$ be an infinite subset of $\mathbb{N}$ and $\left\{S_{N}\right\}_{N \in \mathcal{N}}$ be a family of finite dimension subspaces of $B$ such that $S_{N_{1}} \subset S_{N_{2}}$ if $N_{1}<N_{2}$ and $\cup S_{N}$ is dense in $B$. For all $N \in \mathcal{N}$, let

\footnotetext{
${ }^{1}$ danielinha.dani@gmail.com

${ }^{2}$ gsilva@ibilce.unesp.br

${ }^{3}$ hsilva@ibilce.unesp.br
} 
$f_{N}: S_{N} \rightarrow \mathbb{R}$ be a continuous function that approximates $f(\cdot)$ over $S_{N}$, and let $S_{C, N} \subset S_{N}$ be an approximation of $S_{C}$. Consider the approximated problems family

$$
\left(P_{N}\right) \quad \min _{x \in S_{C, N}} f_{N}(x) \quad N \in \mathcal{N} .
$$

Definition 2.1. Suppose that the functions $f(\cdot)$ and $f_{N}(\cdot)$ and the sets $B, S_{C}, S_{N}$ and $S_{C, N}$ are defined as above.

i) We say $P_{N}$ epi-converge to $P$ if:

a) For all $x \in S_{C}$ there exists a sequence $\left\{x_{N}\right\}_{N \in \mathcal{N}}$, with $x_{N} \in S_{C, N}$, such that $x_{N} \rightarrow^{\mathcal{N}} x$, with $N \rightarrow \infty$, and $\varlimsup \lim _{N}\left(x_{N}\right) \leq f(x)$;

b) For all infinite sequence $\left\{x_{N}\right\}_{N \in \mathcal{K}}, \mathcal{K} \subset \mathcal{N}$, such that $x_{N} \in S_{C, N}$, for all $N \in \mathcal{K}$, and $x_{N} \rightarrow^{\mathcal{K}} x$, with $N \rightarrow \infty$, then $x \in S_{C}$ and $\underline{\lim }_{N \in \mathcal{K}} f_{N}\left(x_{N}\right) \geq f(x)$.

ii) We say the upper semicontinuous functions $\gamma_{N}: S_{C, N} \rightarrow \mathbb{R}$ are optimality functions for the problems $\left(P_{N}\right)$ if $\gamma_{N}(\eta) \leq 0, \forall \eta \in S_{C, N}$ and if $\hat{\eta}_{N}$ is a local minimizer of $\left(P_{N}\right)$ then $\gamma_{N}\left(\hat{\eta}_{N}\right)=0$. We can define the optimality function $\gamma: S_{C} \rightarrow \mathbb{R}$ for $(P)$ in the same way.

iii) The pairs $\left(P_{N}, \gamma_{N}\right)$ of the sequence $\left\{\left(P_{N}, \gamma_{N}\right)\right\}_{N \in \mathcal{N}}$ are consistent approximations to the pair $(P, \gamma)$ if $P_{N}$ epi-converge to $P$ and for all sequence $\left\{x_{N}\right\}_{N \in \mathcal{N}}$ where $x_{N} \in S_{C, N}$ and $x_{N} \rightarrow x \in S_{C}$ we have $\overline{\lim } \gamma_{N}\left(x_{N}\right) \leq \gamma(x)$.

\section{The Impulsive System}

Consider the impulsive system

$$
\left\{\begin{array}{l}
d x=f(x, u) d t+g(x) d \Omega, t \in[0, T] \\
x(0)=\xi^{0} \in \mathcal{C}
\end{array}\right.
$$

where $f: \mathbb{R}^{n} \times \mathbb{R}^{m} \rightarrow \mathbb{R}^{n}$ is linear in $u, g: \mathbb{R}^{n} \rightarrow \mathcal{M}_{n \times q}$, where $\mathcal{M}_{n \times q}$ is the space of $n \times q$ matrices whose entries are real, $\mathcal{C} \subset \mathbb{R}^{n}$ is closed and convex, the function $u:[0, T] \rightarrow \mathbb{R}^{m}$ is Borel measurable and essentially bounded, $\Omega:=\left(\mu,|\nu|, \psi_{t_{i}}\right)$ is the impulsive control, where the first component $\mu$ is a vectorial Borel measure with range in a convex, closed cone $K \subset \mathbb{R}_{+}^{q}$. The second component is such that there exists $\mu_{N}:[0, T] \rightarrow K$ so that $\left(\mu_{N},\left|\mu_{N}\right|\right) \rightarrow^{*}(\mu, \nu)$. As $K \subset \mathbb{R}_{+}^{q}$ we must have $\nu=|\mu|$. The functions $\psi_{t_{i}}:[0,1] \rightarrow K$ are associated to the measure atoms, that is, $\left\{\psi_{t_{i}}\right\}_{i \in \mathcal{I}}$ where $\mathcal{I}$ is the set of atomic index of the measure $\mu$ and we define $\Theta:=\left\{t_{i} \in[0, T]: \mu\left(t_{i}\right) \neq 0\right\}$, where $\mu(t)$ is the vectorial value of the measure in $K$. They are measurable, essentially bounded and satisfy

$$
\begin{aligned}
& \text { i) } \quad \sum_{j=1}^{q}\left|\psi_{t_{i}}^{j}(\sigma)\right|=|\mu|\left(t_{i}\right) \text { a.e. } \sigma \in[0,1] \text {; } \\
& \text { ii) } \int_{0}^{1} \psi_{t_{i}}^{j}(s) d s=\mu^{j}\left(t_{i}\right), \quad j=1,2, \ldots, q,
\end{aligned}
$$

for all $t_{i} \in \Theta$.

The functions $\psi_{t_{i}}(\cdot)$ are given us information about the measure $\mu$ during the atomic time $t_{i} \in \Theta$. 


\subsection{The Reparametrized Problem}

Firstly, we study the impulsive system given by (3). For this, let $\Omega=\left(\mu, \nu,\left\{\psi_{t_{i}}\right\}_{t_{i} \in \Theta}\right)$ be an impulsive control and an arbitrary vector $\xi^{0} \in \mathbb{R}^{n}$. Denote by $\mathcal{X}_{t_{i}}\left(\cdot ; \xi^{0}\right)$ the solution to the system

$$
\left\{\begin{array}{l}
\dot{\mathcal{X}}_{t_{i}}(s)=g\left(\mathcal{X}_{t_{i}}(s)\right) \psi_{t_{i}}(s), s \in[0,1] \\
\mathcal{X}_{t_{i}}(0)=\xi^{0}
\end{array}\right.
$$

Consider

$$
x_{\vartheta}:=\left(x(\cdot),\left\{\mathcal{X}_{t_{i}}(\cdot)\right\}_{t_{i} \in \Theta}\right),
$$

where $\vartheta:=(u, \Omega), x(\cdot):[0, T] \rightarrow \mathbb{R}^{n}$ is a function of bounded variation with the discontinuity points in the set $\Theta$ and $\left\{\mathcal{X}_{t_{i}}(\cdot)\right\}_{t_{i} \in \Theta}$ is the collection of Lipschitz functions defined above.

According to [3], there exists $(\theta(\cdot), \phi(\cdot))$ the graph completion of the measure $\mu$.

Definition 3.1. Let

$$
y(s):= \begin{cases}x(\theta(s)) & \text { if } s \in[0,1] \backslash\left(\cup_{i \in \mathcal{I}} I_{i}\right), \\ \mathcal{X}_{t_{i}}\left(\alpha_{t_{i}}(s)\right) & \text { if } s \in I_{i}, \text { for some } i \in \mathcal{I} .\end{cases}
$$

Then $y_{\vartheta}:=y$ is a reparametrized solution of $(3)$ since $y(\cdot)$ is Lipschitz in $[0,1]$ and satisfies

$$
\left\{\begin{array}{l}
\dot{y}(s)=f(y(s), u(\theta(s))) \dot{\theta}(s)+g(y(s)) \dot{\phi}(s) \text { a.e. } s \in[0,1], \\
y(0)=\xi^{0} .
\end{array}\right.
$$

The next theorem is proved by [4].

Theorem 3.1. Suppose that the impulsive control $\Omega$ is given and $x_{\vartheta}$ is as defined in (4). Then, $y_{\vartheta}$ is a reparametrized solution of (3) if and only if $x_{\vartheta}$ is a solution of (3).

\section{The Impulsive Optimal Control Problem}

Denote by $L_{2}^{m}[0, T]$ the set of all functions defined from $[0, T]$ to $\mathbb{R}^{m}$ that have integrable square.

Let $\beta_{\max } \in(0,+\infty)$ be such that every control $u$ belongs to the ball $B\left(0, \beta_{\max }\right):=$ $\left\{u \in \mathbb{R}^{m} ;\|u\|_{\infty} \leq \beta_{\max }\right\}$.

Define

$$
\hat{\mathcal{U}}:=\left\{u \in L_{\infty, 2}^{m}[0, T] ;\|u\|_{\infty} \leq \omega \beta_{\max }\right\},
$$

where $\omega \in(0,1)$ and $L_{\infty, 2}^{m}[0, T]$ is the set of all functions defined from $[0, T]$ to $\mathbb{R}^{m}$ that are essentially bounded. We consider the $L_{2}$ norm over it.

Now, we define the set of constraints of the control $u$ by

$$
\mathcal{U}:=\left\{u \in \hat{\mathcal{U}} ; u(t) \in \overline{\mathcal{U}} \subset B\left(0, \omega \beta_{\max }\right) \text { a.e. } t \in[0, T]\right\},
$$

where $\overline{\mathcal{U}} \subset \mathbb{R}^{m}$ is a convex, compact subset of the ball $B\left(0, \omega \beta_{\text {max }}\right)$. 
Consider the impulsive optimal control problem

$$
\begin{array}{ll}
\min & f^{0}(x(0), x(T)) \\
& d x=f(x, u) d t+g(x) d \Omega \text { a.e. } t \in[0, T] \\
& x(0) \in \mathcal{C}, u \in \mathcal{U}, \operatorname{gcsup}_{t \in[0, T]}|x(t)| \leq L,
\end{array}
$$

where $f^{0}: \mathbb{R}^{n} \times \mathbb{R}^{n} \rightarrow \mathbb{R}$ is continuous, $L>0$ is given and the other functions and sets are defined as above. Here,

$$
\operatorname{gc} \sup _{t \in[0, T]}|x(t)|=\sup _{s \in[0,1]}|y(s)|
$$

Assumption 1. a) The functions $f(\cdot, \cdot)$ and $g(\cdot)$ are $C^{1}$, and there exist constants $K^{\prime}, K^{\prime \prime} \in$ $\left[1, \infty\left[\right.\right.$ such that, for all $x, \hat{x} \in \mathbb{R}^{n}$ and $u, \hat{u} \in B\left(0, \beta_{\max }\right)$ we have

$$
|f(x, u)-f(\hat{x}, \hat{u})| \leq K^{\prime}[|x-\hat{x}|+|u-\hat{u}|], \quad\|g(x)-g(\hat{x})\| \leq K^{\prime \prime}|x-\hat{x}|,
$$

b) The function $f^{0}(\cdot, \cdot)$ and its first derivative are Lipschitz and is $C^{1}$ over bounded set.

c) The impulsive system given by

$$
\begin{aligned}
& d x=f(x, u) d t+g(x) d \Omega \text { a.e. } t \in[0, T], \\
& x(0)=\xi^{0} \in \mathcal{C}, u \in \mathcal{U}, g c \sup _{t \in[0, T]}|x(t)| \leq L,
\end{aligned}
$$

where all the variables are like above, is controllable.

We define the set of constraints of the control $u \circ \theta$ by

$$
\mathcal{U}_{C}:=\left\{\hat{u} \in \hat{\mathcal{U}}_{1} ; \hat{u}(s) \in \overline{\mathcal{U}} \subset B\left(0, \omega \beta_{\max }\right) \text {, a.e. } s \in[0,1]\right\}
$$

where $\beta_{\max }, \overline{\mathcal{U}}$ and $\omega$ are the same and $\hat{\mathcal{U}}_{1}:=\left\{\hat{u} \in L_{\infty, 2}^{m}[0,1] ;\|\hat{u}\|_{\infty} \leq \omega \beta_{\max }\right\}$.

Define

$$
S_{C}:=\left\{\eta \in \mathcal{C} \times \mathcal{U}_{C} \times \mathcal{P}: \sup _{s \in[0,1]}\left|y^{\eta}(s)\right| \leq L\right\},
$$

whose $\mathcal{U}_{C}$ is as defined above and $\mathcal{P}$ is the set of all $\Omega:=\left(\mu,|\nu|,\left\{\psi_{t_{i}}\right\}\right)$ that satisfies the assumptions of the system (3). We represent by $y^{\eta}(\cdot)$ the solution of the system (6) for each $\eta \in S_{C}$.

We obtain the following reparametrized problem

$$
\left(P_{r e p}\right) \min _{\eta \in S_{C}} f^{0}\left(y^{\eta}(0), y^{\eta}(1)\right) .
$$

As $(P)$ and $\left(P_{r e p}\right)$ has the same solution, up to reparametrization, we will use the consistent approximations in $\left(P_{\text {rep }}\right)$. 


\section{Approximated Problems}

We need a metric over the space $S_{C}$. Consider $\Omega_{1}=\left(\mu_{1},\left|\mu_{1}\right|,\left\{\psi_{t_{i}}^{1}\right\}\right), \Omega_{2}=\left(\mu_{2},\left|\mu_{2}\right|,\left\{\psi_{t_{i}}^{2}\right\}\right) \in$ $\mathcal{P}$. We need to define a metric in the measures space $\mathcal{P}$. Consider the metric given by

$$
d_{3}\left(\Omega_{1}, \Omega_{2}\right)=d_{4}\left(\Omega_{1}, \Omega_{2}\right)+d_{5}\left(\Omega_{1}, \Omega_{2}\right),
$$

where $d_{4}(\cdot, \cdot)$ is a metric given by $[5]$,

$$
\begin{aligned}
d_{4}\left(\Omega_{1}, \Omega_{2}\right) & =\left|\left(\mu_{1},\left|\mu_{1}\right|\right)[0, T]-\left(\mu_{2},\left|\mu_{2}\right|\right)[0, T]\right| \\
& +\int_{0}^{T}\left|F_{1}\left(t ;\left(\mu_{1},\left|\mu_{1}\right|\right)\right)-F_{2}\left(t ;\left(\mu_{2},\left|\mu_{2}\right|\right)\right)\right| d t+\max _{s \in[0,1]}\left|\phi_{1}(s)-\phi_{2}(s)\right|,
\end{aligned}
$$

and $d_{5}(\cdot, \cdot)$ is related to the graph-convergence given by $[6]$,

$$
d_{5}\left(\Omega_{1}, \Omega_{2}\right)=\int_{0}^{1}\left|\dot{\theta}_{1}(s)-\dot{\theta}_{2}(s)\right| d s+\int_{0}^{1}\left|\dot{\phi}_{1}(s)-\dot{\phi}_{2}(s)\right| d s .
$$

Note that $S_{C} \subset \mathbb{R}^{n} \times L_{\infty, 2}^{m}[0,1] \times \mathcal{P}=: B$. Define $d=d_{1}+d_{2}+d_{3}$ the metric over $B$, where $d_{3}$ is given above and

$$
d_{1}\left(\xi^{0}, \xi^{1}\right)=\left|\xi^{0}-\xi^{1}\right|_{\mathbb{R}^{n}} \text { and } d_{2}\left(u_{1}, u_{2}\right)=\int_{0}^{1}\left|u_{1}-u_{2}\right|_{\mathbb{R}^{m}}^{2} d s .
$$

We want to get consistent approximations to the problem $\left(P_{r e p}\right)$. For this, define the sets

$$
\mathcal{N}:=\left\{2^{k}\right\}_{k=1}^{\infty} \text { and } S_{N}:=\mathcal{C}_{N} \times L_{N}^{m} \times \mathcal{P}_{N} \text { for all } N \in \mathcal{N},
$$

where $\mathcal{C}_{N}:=\mathbb{R}^{n} \forall N \in \mathcal{N}$,

$$
L_{N}^{m}:=\left\{u_{N} \in L_{\infty, 2}^{m}[0,1] ; u_{N}(s)=\sum_{k=0}^{N-1} u_{k} \tau_{N, k}(s)\right\},
$$

with $u_{k} \in \mathbb{R}^{m}$ and

$$
\tau_{N, k}(s):= \begin{cases}1 & \forall s \in[k / N,(k+1) / N[\text { if } k \leq N-2, \\ 1 & \forall s \in[k / N,(k+1) / N] \text { if } k=N-1, \\ 0 & \text { otherwise }\end{cases}
$$

and $\mathcal{P}_{N}$ is given by

$$
\mathcal{P}_{N}:=\left\{\left(\mu_{N},\left|\mu_{N}\right|, 0\right): \mu_{N}([0, t]):=F_{N}(t)\right\},
$$

where $\left|\mu_{N}\right|$ is the variation of the measure $\mu_{N}, F_{N}(0)=0$ and over $\left.] 0, T\right]$

$$
F_{N}(t):=\sum_{k=0}^{N-1} \bar{\tau}_{N, k}(t)
$$


and

$$
\bar{\tau}_{N, k}(t):=\left\{\begin{array}{c}
b_{k}+\frac{t-\bar{t}_{k}}{\bar{t}_{k+1}-\bar{t}_{k}}\left(b_{k+1}-b_{k}\right), \forall t \in\left[\bar{t}_{k}, \bar{t}_{k+1}\right], \\
k=0, \ldots, N-1,0=\bar{t}_{0}<\ldots<\bar{t}_{N}=T, \\
0 \quad \text { otherwise }
\end{array}\right.
$$

with $b_{k} \in K$ for all $k=0, \ldots, N-1$. Note that $\mu_{N}$ is an absolutely continuous measure from $[0, T]$ to $K$ ( $K$ is convex) for all $N \in \mathcal{N}$. Furthermore, the graph completion of $\mu_{N}$ is defined by $\theta_{N}:[0,1] \rightarrow[0, T]$ and $\phi_{N}:[0,1] \rightarrow K$ as

$$
\begin{gathered}
\theta_{N}(s):=\bar{t}_{k}+\frac{s-s_{k}}{h}\left(\bar{t}_{k+1}-\bar{t}_{k}\right) \text { whenever } s \in\left[s_{k}, s_{k+1}\right], \\
\phi_{N}(s):=F_{N} \circ \theta_{N}(s),
\end{gathered}
$$

where $h=1 / N, s_{k}=k h$ and $k=0, \ldots, N-1$, and it should satisfies:

i) There exists a constant $b>0$ so that $\theta_{N}(\cdot)$ is Lipschitz of rank $b$ for all $N \in \mathcal{N}$;

ii) There exists a constant $r>0$ so that $\lim \sup _{N \rightarrow \infty}\left\|\dot{\phi}_{N}(\cdot)\right\|_{\infty} \leq r$.

All the results enunciated from here are proved in [7].

Lemma 5.1. $\cup \mathcal{P}_{N}$ is dense in $\mathcal{P}$.

By the density of the union of each set, it follows that $\cup S_{N}$ is dense in $B$.

Given $\eta=\left(\xi_{N}^{0}, u_{N}, \Omega_{N}\right) \in S_{N}$, we can use the Euler's descretization to get the discrete dynamic below by the continuous dynamic given by (6). In this way, take $N \in \mathcal{N}, h=1 / N$ the step size and $s_{k}=k h, k=0, \ldots, N$. We have

$$
\begin{aligned}
y_{N}^{\eta}\left(s_{k+1}\right)-y_{N}^{\eta}\left(s_{k}\right)= & f\left(y_{N}^{\eta}\left(s_{k}\right), u_{N}\left(s_{k}\right)\right)\left(\theta_{N}\left(s_{k+1}\right)-\theta_{N}\left(s_{k}\right)\right) \\
& +g\left(y_{N}^{\eta}\left(s_{k}\right)\right)\left(\phi_{N}\left(s_{k+1}\right)-\phi_{N}\left(s_{k}\right)\right), k=0, \ldots, N-1, y_{N}^{\eta}(0)=\xi_{N}^{0},
\end{aligned}
$$

where $\theta_{N}:[0,1] \rightarrow[0, T]$ and $\phi_{N}:[0,1] \rightarrow K$ are as defined in $\mathcal{P}_{N}$.

Define

$$
S_{C, N}:=\left\{\eta_{N} \in \tilde{S}_{C, N}:\left|y_{N}^{\eta_{N}}(s)\right| \leq L+1 / N \quad \forall s \in[0,1]\right\},
$$

where $y_{N}^{\eta_{N}}(\cdot)$ is given by the linear interpolation of the points $y_{N}^{\eta_{N}}\left(s_{k}\right), k=0, \ldots, N$.

Theorem 5.1. $S_{C, N} \rightarrow^{\mathcal{N}} S_{C}, N \rightarrow \infty$.

Then, we get the approximated problems

$$
\left(P_{r e p}^{C, N}\right) \min _{\eta \in S_{C, N}} f_{N}^{0}\left(y_{N}^{\eta}(0), y_{N}^{\eta}(1)\right) \text { where } f_{N}^{0}\left(y_{N}^{\eta}(0), y_{N}^{\eta}(1)\right):=f^{0}\left(\xi_{N}^{0}, y_{N}^{\eta}(1)\right) .
$$

Theorem 5.2. Suppose that Assumption 1 holds. Let

$$
\gamma(\eta):=\min _{\bar{\eta} \in S_{C}}\left(\left\langle\nabla f^{0}(\xi), \bar{\xi}-\xi\right\rangle+\frac{1}{2} \bar{d}\left(\left(\xi^{0}, u, \Omega\right),\left(\bar{\xi}^{0}, \bar{u}, \bar{\Omega}\right)\right)\right),
$$

with $\xi:=\left(\xi^{0}, y^{\eta}(1)\right), \bar{\xi}:=\left(\bar{\xi}^{0}, y^{\bar{\eta}}(1)\right), \bar{d}=d_{1}+d_{2}+d_{4}$ and $\gamma: S_{C} \rightarrow \mathbb{R}$. Then $\gamma$ is an optimality function to the problem $\left(P_{\text {rep }}\right)$. We can define $\gamma^{C, N}: S_{C, N} \rightarrow \mathbb{R}$ in same way we just defined $\gamma(\cdot)$ and have that $\gamma^{C, N}$ is an optimality function to the problem $\left(P_{\text {rep }}^{C, N}\right)$. 
Theorem 5.3. Suppose that Assumption 1 holds. Then, $\left\{\left(P_{\text {rep }}^{C, N}, \gamma^{C, N}\right)\right\}_{N \in \mathcal{N}}$ is a sequence of consistent approximations to the pair $\left(P_{r e p}, \gamma\right)$.

Theorem 5.4. Let $\left(P_{\text {rep }}^{C, N}\right)$ and $\left(P_{\text {rep }}\right)$ be defined as before. Let $\left\{\eta_{N}\right\}_{N \in \mathcal{N}}$ be a sequence of local (respectively, global) minimizers of $\left(P_{\text {rep }}^{C, N}\right)$ such that $\eta_{N} \rightarrow^{d} \eta$, with $N \rightarrow \infty$ and $\eta \in S_{C}$. Then $\eta$ is a local (respectively, global) minimizer of $\left(P_{\text {rep }}\right)$ and there exists $\mathcal{K} \subset \mathcal{N}$ such that $f_{N}^{0}\left(\xi_{N}^{0}, y_{N}^{\eta_{N}}(1)\right) \rightarrow f^{0}\left(\xi^{0}, y^{\eta}(1)\right)$, with $N \rightarrow \infty, N \in \mathcal{K}$.

Suppose $\left\{\eta_{N}\right\}_{N \in \mathbb{N}} \subset S_{C, N}$ is a sequence of global minimizers of $\left(P_{r e p}^{C, N}\right)$ that is converging to $\eta \in S_{C}$ in the metric $d$. By Theorem 5.4, $\eta$ is a global minimizer of $\left(P_{\text {rep }}\right)$. Then $y^{\eta}(\cdot)$ given by $(6)$ is the function that minimizes $\left(P_{r e p}\right)$. From $y^{\eta}(\cdot)$ is possible to find $x(\cdot)$ that minimizes $(P)$.

\section{Conclusions}

This work aims to contribute with the presentation of the Euler's method application for impulsive optimal control problems. We are contributing with the literature because there are not many works about approximation in the impulsive optimal control case.

\section{Support}

Grant 2011/14121-9, 2014/05558-2 and 13/07375-0, São Paulo Research Foundation (FAPESP).

\section{References}

[1] E. Polak. Optimization, volume 124. Springer-Verlag, New York, 1997.

[2] E. Polak. On the use of consistent approximations in the solution of semi-infinite optimization and optimal control problems. Math. Programming, 62:385-414, 1993.

[3] G. Dal Maso and F. Rampazzo. On systems of ordinary differential equations with measures as controls. 4:739-765, 1991.

[4] P. R. Wolenski and S. Žabić. A differential solution concept for impulsive systems. Dyn. Contin. Discrete Impuls. Syst. Ser. A Math. Anal., 13B:199-210, 2006.

[5] D. Yu. Karamzin. Necessary conditions of the minimum in an impulsive optimal control problem. Journal of Mathematical Sciences, 139:7087-7148, 2006.

[6] P. R. Wolenski and S. Žabić. A sampling method and approximation results for impulsive systems. SIAM J. Control Optim., 46:983-998 (electronic), 2007.

[7] D. Porto. Approximation to Impulsive Optimal Control Problem and Minimum Time Problem on Stratified Domains. Tese de Doutorado em matemática, Unesp-Campus de São José do Rio Preto, 2016. 